\title{
Aux origines de la « science des examens » (1920-1940)
}

The origins of the "science of examinations", 1920-1940

$\mathrm{Zu}$ den Anfängen der „Examenswissenschaft", 1920-1940

Jérôme Martin

\section{(2) OpenEdition}

Journals

Édition électronique

URL : https://journals.openedition.org/histoire-education/817

DOI : $10.4000 /$ histoire-education. 817

ISSN : 2102-5452

Éditeur

ENS Éditions

Édition imprimée

Date de publication : 1 mai 2002

Pagination : 177-199

ISBN : 2-7342-0923

ISSN : 0221-6280

\section{Référence électronique}

Jérôme Martin, « Aux origines de la « science des examens » (1920-1940) », Histoire de l'éducation [En ligne], 94 | 2002, mis en ligne le 08 janvier 2009, consulté le 21 septembre 2021. URL : http:// journals.openedition.org/histoire-education/817 ; DOI : https://doi.org/10.4000/histoire-education.817

Ce document a été généré automatiquement le 21 septembre 2021.

(c) Tous droits réservés 


\title{
Aux origines de la « science des examens » (1920-1940)
}

\author{
The origins of the "science of examinations", 1920-1940 \\ Zu den Anfängen der „Examenswissenschaft“, 1920-1940
}

Jérôme Martin

1 L'historiographie de l'éducation s'intéresse de plus en plus à l'histoire des pratiques pédagogiques, parmi lesquelles les travaux d'élèves et les méthodes d'évaluation tiennent une place importante ${ }^{1}$. Dans cette perspective, les origines de la docimologie, ou "science des examens " $^{2}$ méritent un intérêt particulier. Cette «science ", dont le nom a été forgé par Henri Piéron à partir de deux mots grecs, dokimé, épreuve, et logos, science, se propose d'étudier l'organisation des examens, leurs contenus et leurs objectifs pédagogiques, les méthodes de correction des épreuves ainsi que le comportement des examinateurs et des examinés. Pourtant, l'histoire de la docimologie n'a donné lieu jusqu'à ce jour à aucune étude.

2 Cette discipline a été fondée au début des années 1920 par deux scientifiques français de grand renom, Henri Piéron ${ }^{3}$ et Henri Laugier ${ }^{4}$. Pourquoi et comment, à partir de quels matériaux conceptuels et à partir de quelles expériences en arrivèrent-ils à s'interroger sur la validité des examens? Quelles finalités assignaient-ils à cette « science » et pour quels usages? Pour y répondre, il convient de s'interroger d'abord sur le cheminement intellectuel de ces deux scientifiques.

3 Les origines de la docimologie s'inscrivent aussi dans l'histoire plus large de la psychologie appliquée, c'est-à-dire de la psychotechnique. Le plus souvent, les études consacrées à l'histoire de la psychotechnique accordent une place prépondérante à ses applications au travail industriel. Pourtant, psychologues et physiologistes affirmaient que « la psychotechnique doit comprendre une branche consacrée à l'étude critique des procédés classiques de sélection fondés sur les examens et concours d'ordre scolaire $»^{5}$. Pourquoi cette extension relativement précoce de la psychotechnique aux examens et au champ éducatif, d'autant plus significative qu'elle ne se retrouve pas comme telle dans les autres pays? Faut-il y voir une spécificité française et supposer l'existence 
d'enjeux non strictement scientifiques? Cette liaison précoce entre psychologie appliquée et éducation donne en tout cas à penser que les origines de la docimologie ne peuvent pas être uniquement saisies sous l'angle de l'histoire de la psychologie du travail.

Enfin, la mise en place d'évaluations à trois étapes de la scolarité (CE2, sixième et seconde) entre 1989 et 1992 invite à s'interroger sur les origines d'un pareil dispositif dans l'enseignement général. À l'origine de ces méthodes d'évaluation, Lucie Tanguy et Françoise Roppé ont avancé comme hypothèse l'importation de nouveaux concepts et de nouvelles pratiques venus des États-Unis, en soulignant leur utilisation précoce dans l'enseignement technique ${ }^{6}$. Elles ne citent en revanche ni la docimologie, ni l'Institut national d'étude du travail et d'orientation professionnelle ${ }^{7}$. Dans tous les cas, se pose le problème de la diffusion de ces nouvelles méthodes pédagogiques au sein du système éducatif : celui-ci est-il resté hostile aux innovations pédagogiques? Ces innovations se sont-elles cantonnées dans des secteurs marginaux?

On le voit, les questions suscitées par la docimologie sont nombreuses. Il ne s'agit pas ici d'y apporter des réponses, mais plutôt d'avancer quelques pistes de réflexion à partir de recherches menées sur l'histoire de l'orientation professionnelle.

\section{Psychologie expérimentale et éducation}

6 La science nouvelle "inventée » au début des années 1920 par Henri Piéron et Henri Laugier, s'inscrit dans deux problématiques qui se font écho et se renforcent l'une l'autre. Depuis les années 1880, la psychologie s'autonomise comme discipline scientifique à part entière sur le modèle des sciences expérimentales ${ }^{8}$. Parallèlement, des courants pédagogiques nouveaux, qui s'appuient en grande partie sur les applications éducatives de la psychologie, se développent pendant cette période. Ils s'incarnent au début des années 1920 dans la création de la Ligue internationale d'éducation nouvelle.

\section{La constitution d'une nouvelle science : la psychologie expérimentale}

7 À partir des années 1880 , la psychologie expérimentale se constitue en science, en élaborant un projet scientifique dont l'objet est l'étude de « l'adaptation de l'individu à son milieu ${ }^{9}$, c'est-à-dire de l'individu dans ses activités. Héritière de la physiologie, elle s'en émancipe progressivement avec la création de laboratoires, dont le premier a été celui d'Alfred Binet ${ }^{10}$. Cependant, psychologie et physiologie restent encore longtemps des disciplines voisines. La psychologie se propose de comprendre le fonctionnement des facultés psychologiques qui sont mobilisées par les individus dans les différentes activités humaines, mais aussi les différences d'efficacité et de réussite observables d'un individu à un autre. Dès ses débuts, la psychologie s'intéresse avec Alfred Binet aux «différences individuelles » et à « la recherche de méthodes précises permettant de caractériser les individus et de confronter les différences en les chiffrant». La préoccupation "de rendre plus objectif et plus scientifique l'examen psychologique $»^{11}$ est commune à Alfred Binet et à Édouard Toulouse. 
8 La psychologie expérimentale se dote d'un concept central, celui d'aptitudes, et d'une méthode qui lui est propre, celle des tests. Édouard Claparède définit l'aptitude comme "ce qui différencie, sous le rapport du rendement, le psychisme des individus ${ }^{12}$. Quant aux tests, ce sont des épreuves standardisées qui peuvent être effectuées en laboratoire, mais aussi par simples questionnaires que les sujets complètent. Ils constituent une méthode qui permet à la fois de "caractériser un individu d'un point de vue déterminé » et de le "classer dans un groupe composé d'individus qui lui soient comparables $»^{13}$. Les différences mesurées par les tests et rapportées à un groupe de référence permettent en particulier de définir des aptitudes. Chaque individu se trouve ainsi classé dans une échelle de rendement construite pour chaque type d'aptitude. Tests et aptitudes sont donc indissociables, les premiers fournissant l'instrument de mesure et de construction des secondes. Trois grands types de test ont été progressivement mis au point: le test de développement, dont le modèle est le test Binet-Simon qui «mesure l'évolution mentale »; le test d'aptitudes proprement dit, qui "révèle certaines dispositions naturelles"; enfin, le test de connaissances, qui "permet d'explorer l'acquis scolaire d'un élève, soit en général, soit en une matière particulière $»^{14}$. Avec les tests de développement qui doivent faciliter le repérage des retardés scolaires, les tests d'aptitudes ont été les plus développés, notamment parce qu'ils sont destinés à un usage pratique dans le cadre de l'orientation et de la sélection professionnelles. L'aptitude étant définie comme une "qualité plus grande de réussite ${ }^{15}$ d'un individu dans une activité, les tests ne hiérarchisent pas : ils classent les individus dans une échelle construite de rendements pour différentes aptitudes.

9 La détermination des aptitudes permet de mettre en valeur deux principes fondamentaux. D'une part, les psychologues observent un principe d'homogénéité selon lequel «des différents caractères psychophysiologiques étudiés, la plupart se répartissent dans une population homogène d'individus selon la loi normale de Gauss en cloche ». Ce principe permet de construire des groupes d'aptitudes définissant des métiers. En effet, "en mesurant chez un même individu un grand nombre de caractères, on les voit se répartir encore selon une loi proche de la normale avec seulement une dispersion un peu moindre que celle qu'on rencontre lorsqu'on mesure le même caractère sur une population d'individus différents. On peut penser qu'une personne apte à un métier ne l'est pas nécessairement à tous les autres, que cependant elle sera, en règle générale, apte à tout un groupe de métiers, et non pas uniquement à un seul $\aleph^{16}$. Il convient de souligner à ce propos que les psychologues et physiologistes utilisent très tôt les instruments statistiques ${ }^{17}$. Un second principe, fondamental pour la docimologie, est celui de «l'éducabilité ou la perfectibilité des aptitudes », c'est-à-dire «la propriété qu'ont des fonctions mentales, des opérations, des aptitudes, de se développer par l'exercice $\aleph^{18}$. Aussi la détermination des aptitudes apparait-elle d'emblée comme le fondement scientifique d'une méthode éducative.

10 Cependant, pour comprendre la genèse de la docimologie, il convient d'évoquer plus longuement le rôle de deux fondateurs de la psychologie française, Alfred Binet et Édouard Toulouse.

\section{Binet et la « mesure du degré d'instruction »}

11 La critique des examens et des pratiques pédagogiques remonte au-delà des premiers travaux de docimologie. À la fin des années 1880, à l'initiative des médecins hygiénistes, 
la question du « surmenage » scolaire est déjà posée. Les programmes encyclopédiques et la longueur des journées scolaires sont dénoncés comme facteur de pathologies telles que la fatigue cérébrale. C'est par ce biais que Binet est amené à s'intéresser au milieu scolaire. Entre 1898, date à laquelle il publie avec Victor Henri La fatigue intellectuelle, et 1906, il dirige ou publie plusieurs travaux sur la question de la fatigue scolaire. Cependant, ce sont ses travaux sur l'intelligence et sa mesure qui le conduisent à remettre en cause les formes d'évaluation pratiquées par les enseignants. La dénonciation du "surmenage " se double alors de la dénonciation de l'examen luimême. Binet est ainsi parmi les premiers à se livrer à une critique psychologique des méthodes pédagogiques traditionnelles.

À la tête du Laboratoire de psychologie physiologique de la Sorbonne à partir de 1894 et surtout du Laboratoire de pédagogie expérimentale de l'école de la rue de la Grangeaux-Belles créé en octobre 1905, il oriente dorénavant l'essentiel de ses recherches vers les questions d'enseignement. La mise au point de son échelle de l'intelligence se veut ainsi un instrument pédagogique au service d'une école qui serait ouverte aux applications de la science ${ }^{19}$. S'interrogeant sur la finalité de l'école et sur les critères permettant de la juger efficace, Binet lui assigne deux objectifs: augmenter «le rendement d'un individu particulier » mais aussi faire bénéficier la collectivité de cette augmentation $^{20}$. Dans cette perspective, la psychologie ne peut que s'intéresser à la question de l'évaluation des élèves, c'est-à-dire à la manière dont l'école évalue son propre travail. Or, selon Binet, les examens pratiqués par le système scolaire présentent deux caractéristiques qui les discréditent: d'une part, ils comportent des épreuves arbitraires et d'autre part, ils ne permettent pas de savoir ce qu'ils évaluent. Binet développe cette critique à propos du certificat d'études. Celui-ci est caractérisé par « le défaut de tous les examens où les questions sont laissées à l'arbitraire du juge [...]. Les difficultés n'en sont point dosées avec rigueur ; il n'y a là aucune méthode ${ }^{21}$. C'est ce que confirment largement les résultats des premières études de docimologie. La nécessité d' " organiser des examens qui soient des mesures du degré d'instruction » conduit à l'élaboration d'une méthodologie de l'examen, avec des tests de résultats respectant deux principes : d'une part une standardisation des épreuves et d'autre part, leur étalonnage ${ }^{22}$. Son collaborateur, l'instituteur Vaney, met ainsi au point, en 1906, "un plan d'examens qui permet de mesurer l'instruction d'un élève, âge par âge, depuis sept ans jusqu'à douze ans $»^{23}$. Cette nouvelle approche des métho-des d'évaluation s'inscrit dans la défense d'une nouvelle pédagogie « qui fait de l'écolier un actif, au lieu de le réduire à n'être qu'un écouteur " ${ }^{24}$, et de l'idéal de "l'école sur mesure $»^{25}$.

\section{Toulouse et la fonction régulatrice de la science}

13 Si Binet peut être considéré comme un précurseur de la docimologie, c'est à Édouard Toulouse $^{26}$ et à ses élèves qu'elle doit sa constitution en discipline. Chef de service à l'Asile de Villejuif, Toulouse est à l'origine de plusieurs laboratoires, dont le Laboratoire de psychologie expérimentale rattaché à l'École pratique des hautes études. Henri Piéron y devient son collaborateur en 1900, avant de succéder à Binet. Henri Laugier rejoint Toulouse à l'hôpital Henri-Rouselle à partir de $1923^{27}$.

Dans le sillage de Toulouse, ces scientifiques partagent une même conception de la fonction sociale de la science. Toulouse est en effet à l'origine de la biocratie, théorie 
selon laquelle «la conduite des peuples, de l'humanité toute entière ne sera organisée rationnellement que lorsqu'on aura réalisé un État où le gouvernement des personnes sera basé sur les sciences de la vie c'est-à-dire une Biocratie ${ }^{28}$. Cet eugénisme «français » assigne donc à la science la mission de réorganiser la société sur des bases à la fois plus justes et plus efficaces. En particulier, la sélection des individus en vue de leur affectation à des fonctions sociales lui paraît fondamentale : «Ce n'est pas à l'âge où l'homme se présente à l'entrée d'une carrière que la sélection peut avoir lieu le plus utilement. À ce moment, les meilleurs dons ont pu ne pas être cultivés comme il aurait fallu. Le seul remède à cette situation injuste et surtout contraire à l'intérêt social se trouve dans l'organisation de l'école unique où tous les enfants - riches ou pauvres devront passer et ne pourront s'élever jusqu'aux degrés supérieurs de l'enseignement qu'en fonction de leurs aptitudes ${ }^{29}$. Ainsi, par un autre cheminement que celui suivi par Binet, les scientifiques formés autour de Toulouse accordent aux questions éducatives une place centrale. La détermination scientifique des aptitudes doit permettre à chaque individu d'occuper la fonction sociale la plus adaptée aussi bien pour lui-même que pour la société. Elle s'impose ainsi comme condition et légitimation de l'école unique ; elle justifie également l'orientation professionnelle.

Les fondateurs de la docimologie ne sont pas uniquement liés par des préoccupations scientifiques communes - la psychologie expérimentale et la physiologie - et par un projet social - la biocratie d'Édouard Toulouse. Ils ont en commun leur intérêt pour l'école. La psychologie, en montrant les spécificités des différents âges de la vie et les lois qui les commandent, rejoint les préoccupations des différents courants pédagogiques qui apparaissent au début du $\mathrm{xx}^{\mathrm{e}}$ siècle. Elle apporte notamment une base scientifique aux « méthodes actives ». Les principaux représentants de l'école française de psychologie expérimentale participent d'ailleurs au mouvement de l'éducation nouvelle. Alfred Binet a montré la voie. Dans son ouvrage Les Idées modernes sur les enfants, il affirme "que la détermination des aptitudes des enfants est la plus grosse affaire de l'enseignement et de l'éducation; c'est d'après leurs aptitudes qu'on doit les instruire, et aussi les diriger vers une profession. La pédologie doit avoir comme préliminaire une étude de psychologie individuelle $»^{30}$. Son successeur Henri Piéron joue un rôle important dans la Ligue internationale d'éducation nouvelle, constituée à Calais en 1920, dont il assure à deux reprises la présidence, entre 1931 et 1933.

La docimologie s'inscrit aussi dans ce mouvement en apportant des arguments scientifiques à la dénonciation des formes classiques d'enseignement. Ses promoteurs n'ont de cesse en effet de réclamer la mise en place de nouvelles méthodes d'évaluation, elles-mêmes fondées sur les pédagogies actives. Cependant, c'est le développement de l'orientation professionnelle qui apparaît comme une des premières applications de ce programme.

\section{Docimologie et orientation professionnelle}

17 Les «inventeurs» de la docimologie participent au développement de l'orientation professionnelle qui prend son essor dans les années $1920^{31}$. Julien Fontègne, un des pionniers de l'orientation professionnelle en France, qualifie l'orientation professionnelle de "psychologie appliquée aux vocations $»^{32}$. L'orientation professionnelle est en effet une des seules applications de la psychologie moderne. Son champ d'intervention est vaste puisqu'elle a " pour but de diriger un individu - le plus 
souvent un adolescent, mais aussi un adulte, un chômeur, un mutilé - vers la profession dans laquelle il a le plus de chances de réussir, parce qu'elle répond le mieux à ses aptitudes psychologiques ou physiques ${ }^{33}$. Pour Piéron ou Laugier, il ne s'agit pas simplement d'affecter la main-d'œuvre en fonction des besoins économiques ${ }^{34}$. Bien au contraire, conformément à l'empreinte scientiste léguée par Toulouse, elle s'intègre à un vaste projet dans lequel la science et ses applications doivent résoudre les problèmes sociaux et constituer un instrument de régulation sociale. Déjà en 1904, dans l'ouvrage-manifeste Technique de Psychologie expérimentale publié par Toulouse, Piéron et Vaschide, l'orientation professionnelle se voit assignée une fonction sociale centrale : « en faisant la part de ce que l'éducation technique peut apporter de développement à des facultés existantes, on arrivera à déceler dans un équilibre mental d'une certaine nature, les germes de ce qu'on pourra scientifiquement appeler la vocation, ou tout au moins l'aptitude, bien qu'elle ne se manifeste pas toujours à celui-là même qui la possède. Et l'instruction des individus pourra être régie par des lois d'utilisation scientifiquement déterminées, et pourra, et particulier, développer rationnellement les tendances utiles. Et surtout, classer les individus suivant leurs aptitudes avec une précision bien autre que celle que peuvent fournir des examens superficiels, des concours ou des circonstances fortuites. Telle est l'œuvre d'utilité sociale que la science psychologique, appliquée à des questions concrètes pourra bientôt hardiment revendiquer $»^{35}$. Quelques années plus tard, en 1912, Toulouse peut préciser ce projet, en affirmant qu' « un jour viendra où le concours consistera en un examen médicopsychologique dans lequel les aptitudes particulières de chaque individu seront cotées, et aucun père de famille ne poussera son enfant vers un métier ou une profession sans l'avoir fait examiner, comme on fait dès maintenant essayer une machine ${ }^{36}$.

L'orientation professionnelle subvertit doublement l'école traditionnelle. Au plan des finalités, il ne s'agit plus seulement de transmettre ou d'instruire, mais de rechercher des aptitudes, afin d'assigner à l'individu une fonction utile à la société et correspondant à ses caractéristiques propres. C'est ainsi qu'Henri Piéron replace l'usage des examens scolaires dans le fonctionnement social, afin d'en dévoiler la fonction réelle : «L'admission à des examens - certificat d'études, brevet, baccalauréat, - ou à des concours - bourses, écoles professionnelles, écoles normales, etc. représente une des formes fondamentales de l'orientation professionnelle, à laquelle on ne prend plus garde parce qu'elle est entrée dans la tradition, bien qu'elle réalise une orientation de caractère impératif, sinon sous la forme positive de l'appel vers tel ou tel groupe de carrières, du moins sous la forme négative de la contre-indication ${ }^{37}$. Examens et concours ne sont donc qu'une des modalités de l'orientation professionnelle, au sens où ils ouvrent à des cursus de formation qui débouchent sur des types précis de profession.

19 Au plan des méthodes, l'orientation professionnelle se propose de substituer aux pratiques pédagogiques traditionnelles des méthodes scientifiquement élaborées, c'està-dire des tests. L'orientation professionnelle remplacerait ainsi les autres procédures d'orientation. Pour Piéron, Laugier ou Toulouse, la mise en œuvre de l'orientation professionnelle préfigure une orientation scolaire dont l'objectif serait avant tout la détermination des aptitudes. En effet, pour ces scientifiques, «tout système d'éducation qui met au premier plan de ses préoccupations le développement intégral de la personnalité de l'enfant doit aboutir à une orientation professionnelle rationnelle. Car la tâche de l'école est de préparer l'enfant à la vie ; et la mission de l'éducateur et, 
plus encore que d'aider l'enfant à conquérir des parchemins, de l'acheminer vers une activité sociale, où il réussira, et où il pourra s'épanouir dans un travail heureux et à rendement élevé ${ }^{38}$. La fondation de l'Institut national d'orientation professionnelle en 1928 s'inscrit dans ce vaste projet. Destiné à former des "orienteurs" selon les méthodes scientifiques, il est aussi un centre de recherche. Dans l'esprit des fondateurs, l'Institut et l'orientation professionnelle constituent les bases institutionnelles destinées à promouvoir les acquis de la méthode expérimentale. Selon Piéron, «le développement de l'orientation professionnelle [...] et de l'emploi de méthodes psychotechniques devait contribuer à une certaine transformation des examens dans le sens d'aiguillages rationnels et à l'introduction de méthodes plus objectives dans l'appréciation des aptitudes ${ }^{39}$. Mais ces orientations scientifiques et éducatives débordent la simple promotion des tests. Elles contribuent aussi à une autre approche de l'éducation et des apprentissages. Ainsi l'élaboration théorique et la diffusion dans l'institution scolaire de fiches d'orientation invitent les instituteurs à ne pas évaluer leurs élèves uniquement sur la base des résultats scolaires ${ }^{40}$. À côté de ceux-ci, ils sont invités à évaluer les aptitudes et à tenir compte des caractéristiques psychologiques des enfants. Les examens et évaluations classiques ne sont pas les seuls critères à partir desquels les élèves sont orientés. Cette approche souligne les manques et insuffisances des notations scolaires tout en proposant des méthodes alternatives. L'orientation professionnelle est donc bien le tronc commun à partir duquel la docimologie et l'orientation scolaire doivent pouvoir se déployer. Plus précisément, la docimologie fournit le chaînon entre l'orientation professionnelle et l'orientation scolaire.

\section{La constitution de la docimologie et ses enjeux socio-éducatifs}

20 C'est en juin 1922 qu'Henri Laugier, Henri Piéron et son épouse effectuent la première enquête fondatrice de la docimologie ${ }^{41}$. Elle porte sur 117 élèves âgés en moyenne de 12 ans et 6 mois, appartenant à trois écoles communales de la Seine. Cette première étude a vu la participation de Piéron, mais les grandes enquêtes postérieures sont produites par Laugier. Si Piéron peut revendiquer la paternité de l'intitulé de la nouvelle science, c'est Laugier qui est le plus actif dans sa construction scientifique et sa légitimation. Cependant, Piéron soutient activement ces travaux ${ }^{42}$. D'une part, il publie dans L'Année psychologique qu'il dirige depuis 1912, les études de docimologie. D'autre part, il inaugure en 1932 dans le Bulletin de l'INOP une «Chronique docimologique » qu'il assure lui-même. Il publie également plusieurs articles sur ces questions dans Pour l'ère nouvelle.

Laugier, nommé au CNAM à la chaire de physiologie du travail créée en 1929, mène plusieurs enquêtes avec l'aide de sa collaboratrice Dagmar Weinberg. C'est dans ce cadre que sont publiées les enquêtes les plus importantes ${ }^{43}$. Le début des années 1930 constitue en effet un tournant pour la docimologie: en 1931, la Fondation Carnegie charge l'Institute of International Education de l'Université de Columbia de mener une enquête internationale sur les conceptions, les méthodes, la technique et la portée pédagogique des examens et concours. Dans un premier temps, les fondateurs de la docimologie ne font pas partie du comité français ${ }^{44}$. Si l'on en croit Piéron, l'hostilité de certains milieux universitaires expliquerait leur absence ${ }^{45}$. Et il précise : "grâce au désistement du doyen Maurain, la docimologie a pu pénétrer dans le comité français en 
la personne de notre collègue Laugier... $»^{46}$. L'intérêt de Laugier pour la docimologie renvoie à son engagement en faveur de l'école unique, remontant aux Compagnons de l'Université nouvelle. Cependant, cet intérêt doit aussi être mis en relation avec ses travaux de biotypologie qu'il mène parallèlement avec Toulouse. La fondation de la Société de Biotypologie par Laugier et Toulouse date de 1933. Ils travaillent alors tous deux à la mise au point d'un " examen total à base biotypologique » afin d'établir « une classification des types humains $»$ reposant sur sept examens complets ${ }^{47}$.

Dans les premières études de docimologie publiées entre 1927 et 1938, les analyses posent les bases d'une compréhension des processus d'apprentissage tout en critiquant les méthodes d'enseignement et d'évaluation traditionnelles. À côté de la dénonciation classique du surmenage et $d u$ bachotage, l'analyse scientifique des examens se développe ainsi dans trois grandes directions.

La première est une critique du caractère arbitraire de l'examen traditionnel, tandis que le concours, lui, ne sélectionne les candidats qu'en vertu du hasard. Le recours aux méthodes statistiques permet aux docimologues de faire apparaître des défauts structurels des examens et concours traditionnels. S'appuyant sur la loi de Gauss, ils contestent l'usage de la notion de moyenne arithmétique. Celle-ci est d'autant plus arbitraire que les critères d'évaluation font intervenir la subjectivité du correcteur. Laugier et Weinberg observent en effet que d'un correcteur à l'autre, les échelles de notes varient. Même si ces correcteurs respectent la répartition de leur notes selon la loi de Gauss, les différences d'échelles de note ont des conséquences sur le résultat final. En effet, « le succès à l'examen étant conditionné par la note moyenne tirée de deux ou plusieurs épreuves, l'examinateur qui use d'une échelle nettement plus limitée dévalorise son épreuve, car son collègue en utilisant les notes extrêmes, fait pencher la moyenne de son côté ${ }^{48}$. Ainsi intervient le "coefficient subjectif»: selon que les examinateurs utilisent ou non toute l'échelle des notes, les résultats finaux en seront affectés. Selon l'étude de Laugier et Weinberg, « dans le cas d'un concours, ce ne seront pas les mêmes candidats qui seront choisis par les deux examinateurs; dans notre cas, la moitié des candidats admis par l'un seraient rejetée par l'autre et vice versa ». Aussi convient-il de former les correcteurs à la correction afin d'éviter de trop fortes disparités d'un examinateur à un autre.

L'étude des rendements scolaires constitue le second axe de réflexion. Les premiers travaux de docimologie sont en effet pionniers dans le domaine de la statistique appliquée au rendement scolaire. L'objectif est de déterminer les caractéristiques psychophysiologiques des élèves examinés. Laugier, Toulouse et Weinberg construisent pour cela une classification scolaire en procédant à un examen biotypologique. À partir d'un échantillon de 125 élèves de cours moyens d'une école primaire de Paris, ils établissent que « les meilleurs élèves sont parmi les plus jeunes, les derniers parmi les plus âgés » et que dans la partie physique du profil, les «meilleurs élèves se montrent, en général, [...] inférieurs ; les mauvais supérieurs à l'ensemble du groupe ». Ainsi sont mis en valeur deux critères - l'âge et la constitution psychique et psychologique - dans l'explication du rendement scolaire. Ces recherches sur le rendement conduisent également Laugier et Weinberg à s'interroger sur "les différences entre les sexes " Examinant les notes obtenues par des étudiants et des étudiantes de la faculté de science de Paris ${ }^{50}$, ils observent d'une part que la réussite des étudiantes est tout à fait comparable à celle des étudiants et que, d'autre part, leur réussite diffère selon les disciplines. Bien qu'ils ne proposent pas d'explication à ces observations, cette étude 
est pionnière dans le domaine de la statistique scolaire et des enquêtes de la sociologie de l'éducation.

Un troisième axe de réflexion concerne la fonction sociale de la sélection et la finalité de l'examen. Les fondateurs de la docimologie soulignent la confusion qui préside à la définition de la finalité des examens et concours. En effet, examens et concours traditionnels mélangent systématiquement deux types d'évaluation. D'une part, il peut s'agir de «contrôler les résultats d'une formation éducative, de vérifier des acquisitions, d'évaluer le bagage de connaissances assimilées et de déterminer si, pour un écolier donné, la tâche d'un enseignement peut être considéré comme achevée ». Ce devrait être la finalité du CEP par exemple. D'autre part, il peut s'agir de « déterminer les aptitudes propres d'enfants ou de jeunes gens, qui devront bénéficier d'une formation éducative particulière ", comme dans le cadre des concours d'entrée dans les écoles. Cette distinction établie entre deux types d'évaluation devrait régir le fonctionnement des examens, mais, note Piéron, «en fait, les deux points de vue interviennent toujours, les connaissances servant bien souvent à déterminer le classement même dans les concours où les aptitudes devraient essentiellement fonder la sélection, les capacités individuelles l'emportant d'autre part aux yeux de certains examinateurs sur l'étendue des connaissances, pour l'évaluation du niveau des candidats à des examens institués pour le contrôle d'une formation éducative ». À cet égard, le baccalauréat fournit le meilleur exemple, puisqu'il certifie les sortants de l'enseignement secondaire tout en déterminant les individus aptes à entrer dans l'enseignement supérieur. La docimologie invite à distinguer deux types d'examens, les uns contrôlant l'acquisition de connaissances, les autres servant à déterminer des aptitudes individuelles.

Cependant, la critique des examens et concours ne signifie pas que les docimologues renoncent à la sélection et à sa fonction sociale. Bien au contraire, ils prennent la mesure de la fonction sociale des diplômes dans les sociétés développées, et particulièrement en France. Henri Piéron souligne d'ailleurs, au début des années 1930, la généralisation du phénomène de certification affectant de nombreuses professions : « Il semble bien difficile dans notre organisation sociale de renoncer à ces sanctions qui garantissent un certain savoir, alors que, de plus en plus, on étend ce contrôle, aux médecins, dentistes, sages-femmes, vétérinaires, avocats, y ajoutant maintenant les architectes, les ingénieurs, en attendant que d'autres bientôt s'y ajoutent encore ${ }^{51}$. D'autre part, les fondateurs de la docimologie ont toujours été partisans de l'orientation et de la sélection des individus. Henri Piéron précise ainsi que l'examen de sélection à l'entrée d'une carrière est "indispensable à une organisation sociale rationnelle, mais à la condition que la sélection se fasse bien sur la base des aptitudes requises, ce qui est loin d'être le cas dans la plupart des concours». La docimologie pose donc le problème de la légitimité de la sélection, c'est-à-dire des critères sur lesquels s'opère l'affectation sociale des individus au travers du tamis du système scolaire. Piéron dénonce l'inégalité foncière qui caractérise le système scolaire: les examens et concours traditionnels affectent des individus sur de fausses évaluations. Réussir un concours ne signifie pas posséder les aptitudes nécessaires à telle ou telle carrière, mais avoir été bien préparé à l'épreuve. Ainsi, « au lieu de pouvoir juger les aptitudes naturelles des individus, on se trouve en présence de candidats dressés aux épreuves, et dont certains réussiront mieux parce que plus habilement dressés, prenant la place d'autres réellement plus intelligents; et l'on reçoit ainsi les poulains des écuries les mieux outillées $\aleph^{52}$. Ce corpus critique étayé par des travaux scientifiques 
remet en cause non seulement les pratiques pédagogiques mais, plus fondamentalement, le principe de la méritocratie revendiquée par l'école républicaine.

\section{L'école unique, la sélection scolaire et la docimologie}

Piéron dresse un bilan mitigé des effets des premiers travaux de docimologie et observe

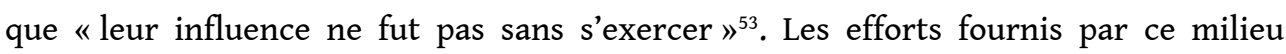
restreint de scientifiques pour constituer et légitimer la docimologie en tant que « science » rencontrent en effet un certain écho, notamment à la faveur du débat sur l'école unique. Comme le souligne Piéron, «l'organisation de l'école unique pose de tous côtés le problème des examens et des méthodes de sélection ${ }^{54}$. Ces questions prennent alors suffisamment d'ampleur pour que les recherches effectuées en matière d'orientation professionnelle depuis le début du siècle soient réinvesties sous un autre angle afin d'en constituer une variante nouvelle et pédagogique. Les années 1920-1930 sont en effet marquées par les débats sur l'école unique et sur la sélection scolaire à l'entrée de l'enseignement secondaire et de l'enseignement supérieur. La docimologie naissante qui repose sur les mêmes fondements théoriques et les mêmes objectifs sociaux trouve là un contexte favorable. Le corpus théorique constitué par les psychologues et physiologistes est d'ailleurs utilisé par certains acteurs du système éducatif. Ainsi, en 1933, le SNI reprend à son compte la distinction entre examens de connaissances et examens d'aptitudes, qu'il intègre à son projet de classe d'orientation entre 11 et 14 ans $^{55}$. Au congrès de Nice, en 1938, il se prononce pour « un système plus scientifique de mesure des connaissances acquises" et "un ensemble d'épreuves étalonnées indiquant, avec une approximation utile, les aptitudes probables de chaque enfant $\|^{56}$. Ces mêmes idées sont reprises au niveau de la Fédération internationale des associations d'instituteurs. À la conférence de Santander, en août 1933, Dumas précise ainsi dans son rapport que «le but des examens est de fournir les éléments d'une répartition rationnelle (orientation) des élèves dans les diverses branches de l'activité humaine ", lesquels examens peuvent « constater des connaissances acquises » ou des "aptitudes à des travaux futurs $»^{57}$. Sans prétendre recenser de manière exhaustive toutes les occasions dans lesquelles les acteurs convoquent la docimologie pour conforter leur argumentation, on peut repérer quelques indices de son influence.

L'apparition de la docimologie en tant que "science" intervient au moment où les premières mesures prises en faveur de l'école unique - même modestes - font apparaître certaines difficultés d'application. En 1924, l'admission gratuite d'élèves dans les classes élémentaires et primaires dans les lycées et collèges ${ }^{58}$ prend ainsi au dépourvu les membres de l'Instruction publique. Parmi les nombreux problèmes que soulèvent les inspecteurs d'académie dans leurs rapports ${ }^{59}$, celui de la sélection mérite d'être souligné. L'inspecteur d'académie du Doubs indique bien les enjeux pédagogiques et sociaux de la réforme : "Là où nous connaîtrons les plus sérieuses difficultés, c'est quand il nous faudra faire une sélection dans le nombre devenu trop grand des demandes d'admission. [...] Et cette sélection indispensable, sur quel principe la fonder en toute justice? Faudra-t-il recourir à un examen? Oui, sans doute ; mais ce procédé de classement qui peut être appliqué à des élèves de $7^{\mathrm{e}}$ et de $8^{\mathrm{e}}$, ne saurait convenir à des enfants plus jeunes. Pour ces derniers, c'est une enquête d'un autre genre que nous devons instituer. À qui nous adresserons-nous alors? Comment parviendrons-nous à 
connaître les aptitudes des enfants, la situation de fortune des parents, leurs intentions, leurs ambitions pour l'avenir de leurs fils? $»^{60}$. Quant au proviseur du lycée de Vesoul, il exprime un certain désarroi face à une tâche étrangère aux pratiques de l'enseignement secondaire. Ayant interrogé les professeurs des classes élémentaires "sur les modalités possibles de la sélection», notamment sur «les facteurs déterminants du choix", il a obtenu des réponses traduisant leur incapacité pédagogique à prendre en charge le problème: "Les professeurs sont unanimes à demander qu'on leur confie des enfants qui, par leur âge et leurs dispositions, sont tels qu'ils puissent être utilement préparés à l'enseignement secondaire ». Le proviseur ne peut que constater son impuissance : pour réaliser une juste sélection il faudrait «que nous connaissions mieux, nous apprenions ce qu'il y a de bon chez le voisin, ce qui convient aux enfants selon leurs aptitudes, la carrière choisie, la situation et les désirs des parents. Il faudrait que nous apprenions à voir dans les enfants, non des numéros qui grossissent nos effectifs, mais des cerveaux à deviner pour les guider dans la voie qui semble être la leur, même si ce n'est pas celle où nous traçons notre ornière ${ }^{61}$. Ainsi, dans un vocabulaire prudent mais évoquant les préoccupations des promoteurs de l'Éducation nouvelle, ce proviseur souligne combien la mise en œuvre de l'École unique appelle une transformation profonde des finalités et des pratiques pédagogiques de l'enseignement secondaire. Mais, du même coup, le certificat d'études primaires est lui aussi déstabilisé dans sa finalité. Dès lors qu'un accès à l'enseignement secondaire des élèves du primaire est envisagé, quelle doit être sa fonction?

Dans le cadre du projet de prolongation de la scolarité obligatoire conçu sous le ministère d'Anatole de Monzie, la fonction du certificat d'études primaires est l'objet d'interrogations. Barrier, directeur-adjoint au ministère, reconnaît ainsi qu' « on s'est trouvé [...] amené nécessairement à envisager deux certificats d'études différents : l'un, qui serait exigé pour l'entrée dans les enseignements de second degré et aussi pour l'accès au second stade des écoles primaires serait un examen de passage ; l'autre, passé à la fin des études primaires, vers 14 ou 15 ans, serait véritablement l'examen de fin d'études primaires ${ }^{62}$. Au cours de sa conférence, Barrier évoque également la nécessité de procéder à la détermination des aptitudes à l'aide de tests, afin d'effectuer une orientation des élèves. Ces réflexions montrent que les préconisations avancées par les partisans de l'Éducation nouvelle et par la docimologie rencontrent un écho dans la perspective de la réalisation de l'école unique. La réforme du certificat d'études, prévue par l'arrêté du 22 mars 1938, bien que non appliquée, s'inspire partiellement de ces idées. Piéron note ainsi que « moins d'importance est donnée à cet examen de contrôle de la formation primaire, plus d'objectivité est assurée, et les épreuves de questions écrites tendent à introduire le principe des méthodes des tests ». Il se félicite « de voir l'examen ramené à un contrôle des capacités élémentaires qui doivent être acquises au cours de l'enseignement du premier degré $"^{63}$.

La réalisation progressive de la gratuité du lycée pose donc la question de la sélection qui doit protéger l'enseignement secondaire. Les réformes menées par le ministère Jean Zay, bien qu'elles ne modifient pas la forme et l'organisation des examens, comportent des éléments inspirés des critiques formulées par les spécialistes de la docimologie. Ainsi, Jean Zay précise dans une circulaire de 1937 à l'intention des examinateurs la fonction du baccalauréat: il "n'est pas le but des études secondaires, il en est seulement la vérification. Le diplôme doit attester, non point que le candidat connaît toutes les parties du programme, mais qu'il est capable, même sur une question qu'il n'a pas directement étudiée, de faire preuve d'intelligence et de méthode ». Il ne s'agit 
plus seulement de contrôler des connaissances mais aussi d'évaluer des capacités et des méthodes. De plus, afin de corriger les travers les plus criants de l'examen, Jean Zay invite à généraliser «la pratique des réunions d'entente entre examinateurs d'une même série d'épreuves écrites » afin d'établir «entre les correcteurs une échelle de notes communes à tous»; pour les épreuves orales, il recommande «de faire mentionner sur le livret scolaire les œuvres ou les textes qui ont été étudiées pendant les deux dernières années ». Ce sont bien les enseignements de l'Éducation nouvelle et de la docimologie qui inspirent ces recommandations. Si elles ne remettent pas en cause, selon lui, le fonctionnement de l'examen et sa nature foncièrement viciée, Henri Piéron les qualifie pourtant d' « observations justes » et de " judicieux conseils » ${ }^{64}$.

31 Les «classes d'orientation " s'inscrivent également dans ce courant en accordant une grande place à la pédagogie active et, surtout, en mettant au centre de la politique scolaire la notion d'orientation. Bien que le ministère n'ait pas introduit officiellement l'usage des tests au sein des classes d'orientation, des professeurs prennent l'initiative de les utiliser directement ou de faire appel à des spécialistes. À Sens, Roger Gal utilise des tests pour déterminer les aptitudes des élèves. À Sèvres, les professeurs font appel à Dagmar Weinberg pour «élaborer des tests qu'on puisse utiliser dans les classes d'orientation ». Plus généralement, nombreux sont les professeurs de ces classes qui constatent «que l'utilisation des tests, qui ne diffèrent que très peu des exercices scolaires habituels, pouvait se faire très facilement $»^{65}$. L'expérience facilite l'utilisation de méthodes nouvelles. L'usage du test comme instrument d'évaluation et de détermination des aptitudes peut ainsi entrer, bien que modestement, dans l'enseignement secondaire. L'expérience acquise dans le domaine de l'orientation professionnelle est également mise au service de ces classes. En septembre 1937, le ministère de l'Éducation nationale organise un stage destiné aux professeurs des classes d'orientation au cours duquel la fiche scolaire d'orientation proposée par Julien Fontègne est adoptée ${ }^{66}$. Ainsi le corpus théorique et pratique élaboré par les promoteurs de l'orientation professionnelle et de la docimologie trouve de nouveaux champs d'application.

L'ouverture des lycées, la "crise des professions libérales » et le contexte général de dépression économique des années 1930 remettent en cause la voie royale que constituaient jusqu'alors le secondaire et le supérieur, suscitant tensions et interrogations. Au début des années 1930, le secrétaire général de la Fédération des associations de parents d'élèves souligne les difficultés nouvelles rencontrées par les familles dans ce nouveau contexte scolaire : «c'est devenu un métier, une profession, une technique, que d'orienter ses enfants vers les carrières où ils ne mourront pas de faim ! On n'a plus le droit d'engager un enfant, fils ou fille, vers les études sans en connaître les voies d'accès et les points d'aboutissement " ${ }^{67}$. Les nouvelles concurrences scolaires renforcent l'enjeu des examens. Le baccalauréat cristallise ces tensions. Ainsi, L'Information universitaire publie en 1933 un article d'un étudiant qui, dénonçant dans les examens de licence un concours masqué, conclut sur la nécessité de "faire la sélection à l'entrée et non à la sortie de l'université ${ }^{68}$. La finalité du baccalauréat est en effet une des questions posées par la docimologie. L'imprécision qui préside aux objectifs de cet examen conduirait en effet nombre d'étudiants vers des impasses. Cette critique est également formulée à propos des études de médecine dont le caractère sélectif apparaît doublement infondé: d'une part, en raison de l'utilisation du baccalauréat et du latin comme critère de sélection; d'autre part, en raison du nombre important d'échecs tout au long de la scolarité. Pour les «docimologues», non 
seulement la sélection est arbitraire et n'apporte aucune garantie sur la qualité des étudiants, mais elle produirait des déclassés sociaux.

L'enseignement secondaire est également l'objet d'études psychotechniques qui ont une dimension docimologique. Au début des années 1930, Charles Dietz, diplômé de l'Institut de psychologie de Paris, effectue une enquête sur la sélection des élèves de l'enseignement secondaire en utilisant le test d'intelligence logique de Jean-Maurice Lahy. Il s'agit, à l'aide de tests, de « déterminer la proportion de jeunes Français dans l'ensemble, de jeunes élèves de chaque ordre d'enseignement en particulier, qui seraient aptes à l'enseignement secondaire ${ }^{69}$. Son étude établit que seuls $10 \%$ des élèves du primaire et $50 \%$ des élèves de lycée sont aptes à l'enseignement secondaire ${ }^{70}$. Cependant, relevant une "corrélation appréciable » entre tests et épreuves scolaires, Dietz se prononce pour une "sélection opérée sur l'avis des instituteurs ou professeurs ${ }^{71}$.

La commission d'orientation professionnelle du Bureau universitaire d'information sur les carrières (BUIC), constituée en 1936, décide en juin 1937 de mener des expériences d'orientation professionnelle dans les lycées. Il s'agit « d'étudier dans quelle mesure les procédés scientifiques d'orientation professionnelle actuellement utilisés pour les jeunes gens de l'enseignement primaire et de l'enseignement technique peuvent s'adapter à des élèves âgés, ayant poursuivi des études secondaires et se destinant à des carrières intellectuelles ». Si cette étude s'applique à tous les niveaux du lycée, elle se concentre particulièrement sur les classes de $6^{\mathrm{e}}$, «gros problème actuel $»^{72}$. On le voit, la docimologie apparaît alors nettement comme l'application des méthodes de la psychotechnique à l'enseignement général, non concerné à cette époque par le dispositif d'orientation professionnelle ${ }^{73}$. La fin des années 1930 constitue une période d'élargissement de la docimologie à l'ensemble du "système éducatif». Les instruments scientifiques, les bases institutionnelles et le milieu scientifique concerné sont suffisamment établis pour envisager, en 1936, une grande enquête sur le niveau intellectuel des enfants de 6 à 12 ans par un test collectif ${ }^{4}$. Cependant, les résistances du secondaire et de sa clientèle rendent ces avancées précaires et en grande partie sans lendemain.

Bien que restée marginale dans la pratique pédagogique des enseignants, la docimologie appartient pleinement au corpus théorique et pratique des courants réformateurs de l'éducation. Ainsi, le plan Langevin-Wallon intègre les principes élaborés par le petit milieu scientifique constitué autour de Piéron et Laugier. Dans les années 1950, les mêmes hommes poursuivent et étendent leurs enquêtes. Si l'enseignement secondaire ne reprend pas à son compte l'usage des tests, les scientifiques entreprennent des travaux importants sur les résultats scolaires des élèves. De son côté, l'enseignement professionnel semble davantage ouvert aux pratiques pédagogiques proposées par la docimologie et la psychotechnique. Les liens originels entre psychotechnique et enseignement technique et professionnel se perpétuent ${ }^{75}$.

Ce rapide survol des origines de la docimologie montre qu'elles mériteraient un examen plus attentif. La docimologie est née sous le double sceau des préoccupations scientifiques d'un groupe restreint de savants qui cherchaient à proposer une forme originale de rationalisation sociale, et de préoccupations socio-éducatives, autour de l'éducation nouvelle et de l'école unique. Elle s'est affirmée à la fois comme une critique et comme une alternative aux pratiques traditionnelles d'évaluation. L'intérêt porté 
par les fondateurs de la docimologie à l'orientation professionnelle montre que ces deux mouvements sont solidaires. Ils reposent sur les mêmes bases conceptuelles, mais plus encore, l'orientation professionnelle en utilisant de nouvelles pratiques d'évaluation dans le primaire et le technique, remet en cause la toute puissance des examens traditionnels. Les années 1920 et 1930 préparent les réformes entreprises par Jean Zay qui s'appuient notamment sur les notions d'orientation et d'aptitudes. Le plan Langevin-Wallon s'inspirera, en partie, des acquis de l'orientation et de la docimologie. Ainsi, même si elle n'a pas débouché en France sur une véritable institutionnalisation, et a échoué notamment à prendre pied dans «l'Empire du milieu », la docimologie a exercé une certaine influence sur les pratiques éducatives dans l'enseignement primaire, technique et professionnel, influence qu'il conviendrait de mesurer. Aussi serait-il utile de la mesurer avec précision et d'identifier les réseaux et les modalités de sa diffusion. Il resterait aussi à évaluer la postérité de la docimologie qui, au regard des évolutions pédagogiques des vingt dernières années, est loin d'être négligeable.

\section{NOTES}

1. Les travaux d'André Chervel ont été novateurs dans ce domaine.

2. Elle se complète par la docimastique, ou technique des examens, et par la doxologie, qui est l'étude systématique du rôle que l'évaluation joue dans l'éducation.

3. Sur Henri Piéron, consulter Maurice Reuchlin : «Henri Piéron », Bulletin de psychologie, t. 237, n 18, pp. 7-9, décembre 1964 ; «Henri Piéron », Christophe Charle (éd.) : Les professeurs du Collège de France, dictionnaire biographique 1901-1939, CNRS-INRP, 1988.

4. Sur Henri Laugier voir William H. Schneider, Henri Laugier: "The Science of work and the workings of science in France, 1920-1940 ", Cahiers pour l'histoire du CNRS, Éditions du CNRS, 1989-5; Jean-Louis Crémieux-Brilhac et Jean-François Picard (dir.): Henri Laugier en son siècle.Cahier pour l'histoire de la recherche, CNRS éditions, 1995 ; "Henri Laugier » in Claudine Fontanon et André Grelon (dir.): Les Professeurs du CNAM, INRP-CNAM, 1994, t. 2, pp. 50-60 ; Chantal Morelle et Pierre Jacob : Henri Laugier, un esprit sans frontières, Bruylant-LGDJ, 1997.

5. H. Piéron, $M^{\text {me }}$ H. Piéron et H. Laugier : «Étude critique de la valeur sélective du certificat d'études et comparaison de cet examen avec une épreuve par tests. Contribution à une docimastique rationnelle", Compte rendu de la Ive Conférence internationale de psychotechnique, Paris, Institut international de coopération intellectuelle, 10 au 10 octobre 1927, Paris, Félix Alcan, 1929, p. 499.

6. Françoise Roppé, Lucie Tanguy (dir.) : Savoirs et compétences. De l'usage de ces notions dans l'école et l'entreprise, L'Harmattan, 1994. Étudiant le «passage des savoirs aux compétences » dans le cas du français, Françoise Roppé identifie «trois filiations intellectuelles » : «la psychopédagogique, la linguistique et la psycholinguistique cognitiviste », p. 64 .

7. Créé en 1928 à l'initiative de Henri Piéron et Julien Fontègne, l'INOP (futur INETOP) est un établissement libre d'enseignement supérieur qui délivre un diplôme de conseiller d'orientation. Il est reconnu par l'État par un décret du 25 juin 1930. Il est rattaché au CNAM en 1941.

8. Sur les débuts de la psychologie en France, consulter Geneviève Paicheler : L'invention de la psychologie moderne, L'Harmattan, 1992; Annick Ohayon: L'impossible rencontre. Psychologie et 
psychanalyse en France, 1919-1969, La Découverte, 1999; Serge Nicolas: Histoire de la psychologie française. Naissance d'une nouvelle science, In Press Editions, 2002.

9. A. Binet : Les Idées modernes sur les enfants (1909), rééd. Flammarion, 1973, p. 27.

10. D'abord nommé en 1892 directeur-adjoint du laboratoire de psychologie physiologique de la Sorbonne par Beaunis, Binet en prend la direction en 1895. Sur Alfred Binet, consulter Guy Avanzini : Alfred Binet, PUF, 1999.

11. H. Piéron : «Les origines, en France, de la méthode des tests et la signification pédagogique de l'œuvre de Binet », Pour l'ère nouvelle. Revue mensuelle d'éducation nouvelle, juillet 1932, p. 164.

12. E. Claparède : L'orientation professionnelle. Ses problèmes et ses méthodes, Bureau international du travail. Études et documents. Série 3 - Enseignement, n 1, Genève, octobre 1922, p. 73.

13. H. Piéron : «La notion d'aptitude en éducation », Pour l'ère nouvelle. Revue mensuelle d'éducation nouvelle, juillet-août 1929, p. 140.

14. R. Duthil : «Les applications pédagogiques de la méthode des tests ", Pour l'ère nouvelle, op. cit., juillet-août 1929, p. 141.

15. H. Piéron : « La notion d'aptitude en éducation », op. cit., p. 137.

16. Encyclopédie française, t. VIII, La vie mentale, 1938, section C : La profession, pp. 84-92.

17. Ainsi, Laugier et Weinberg sont parmi les premiers à utiliser la méthode d'analyse factorielle de Spermann. Bonnardel poursuivra ce type de travail : "Application de la méthode d'analyse factorielle de Thurstone à l'étude de la notation des copies d'examens », Le Travail humain, 1946, pp. 150-167; voir M. Reuchlin : «Contributions à l'histoire des méthodes statistiques employées en psychologie ", Psychologie et Histoire, 2003, vol. 4, pp. 1-60.

18. R. Laufer et G. Paul-Boncour: L'orientation professionnelle du point de vue particulièrement scolaire. La préadaptation scolaire. Rapport à M. le Directeur de l'Enseignement primaire de la Seine, Librairie Delagrave, 1924, p. 36.

19. M. Vial : Les enfants anormaux à l'école. Aux origines de l'éducation spécialisée, 1882-1909, Armand Colin, 1990.

20. A. Binet : Les Idées modernes sur les enfants, op.cit., p. 27.

21. Ibid.,p. 41.

22. " $1^{\circ}$ L'examen n'est pas livré au hasard, au caprice de l'inspiration, aux surprises des associations d'idées, il se compose d'un système de questions dont la teneur est invariable, et dont la difficulté est dosée; $2^{\circ}$ le degré d'instruction d'un enfant n'est point jugé, in abstracto, comme bon, médiocre, mauvais, suivant une échelle subjective de valeur ; il est comparé au degré d'instruction de la moyenne d'enfants de même âge et de même condition sociale qui fréquentent les mêmes écoles ", A. Binet : Les Idées modernes..., op. cit., p. 33.

23. Ibid., p. 33.

24. Ibid., p. 139.

25. Ibid., p. 22.

26. Michel Huteau vient de consacrer un ouvrage à Toulouse : Psychologie, psychiatrie et société sous la Troisième République. La biocratie d'Edouard Toulouse, L'Harmattan, 2002.

27. De 1923 à 1932, il est chef de laboratoire de physiologie appliquée à la prophylaxie mentale.

28. » La conduite de la vie », La Prophylaxie mentale, 17 juin 1929. Cité par A. Drouard: L'eugénisme en questions. L'exemple de l'eugénisme "français ", Ellipses, 1999, p. 40. Sur le mouvement eugéniste et le rôle de Toulouse, voir J.-B. Wojciechowski : Hygiène mentale et hygiène sociale : contribution à l'histoire de l'hygiénisme, L'Harmattan, 1997.

29. Ibid., p. 43.

30. A. Binet : Les Idées modernes sur les enfants, op. cit., p. 21.

31. Le décret du 26 septembre 1922 fixe le premier cadre réglementaire à son développement en plaçant l'orientation professionnelle sous la tutelle du sous-secrétariat à l'Enseignement technique. 
32. J. Fontègne: L'orientation professionnelle et la détermination des aptitudes, Neuchâtel-Paris, Delachaux et Niestlé, 1921, p. 58. Sur Julien Fontègne, consulter la biographie d'André Caroff: Julien Fontègne. Un pionnier de l'orientation professionnelle, INETOP, dactylographié, 125 pages.

33. E. Claparède : L'orientation professionnelle. Ses problèmes et ses méthodes, Bureau international du travail, Études et Documents. Série 3 - Enseignement, n 1, Genève, octobre 1922, p. 20.

34. J. Martin: «L'orientation professionnelle en France, en Allemagne et en Grande-Bretagne dans les années 1920 : un instrument de rationalisation?", Histoire \& Sociétés. Revue européenne d'histoire sociale, $\mathrm{n}^{\circ}$ 2, 2e trimestre 2002, pp. 94-106. Sur le mouvement d'orientation professionnelle, voir André Caroff : L'organisation de l'orientation des jeunes en France. Évolution des origines à nos jours, EAP, 1987.

35. Cité par H. Piéron: «Les origines, en France, de la méthode des tests et la signification pédagogique de l'œuvre de Binet ", art. cit., p. 164

36. Cité par H. Piéron : "La place de l'Institut dans l'histoire de l'orientation professionnelle », Bulletin de l'Institut national d'orientation professionnelle (BINOP), $2^{\mathrm{e}}$ série, $\mathrm{n}^{\circ} 9,1953$, pp. 7-8.

37. H. Piéron : «Orientation professionnelle et Docimologie », BINOP, $\mathrm{n}^{\circ}$ 6, juin 1929, p. 161.

38. H. Laugier, E. Toulouse et D. Weinberg : «La Biotypologie et l'orientation professionnelle » (Extrait de la communication présentée au Congrès de Nice), Pour l'ère nouvelle, novembre 1933, p. 249.

39. H. Piéron : Examens et Docimologie, P.U.F., 1963, p. 27.

40. En 1930, Henri Piéron préconise un modèle de « fiche psychopédagogique d'orientation pour les éducateurs » qu'il publie dans le BINOP, n², février 1930, pp. 29-35.

41. Les résultats de cette enquête sont publiés dans L’Année psychologique (t. XXIII, pp. 146-175) et sont l'objet d'une communication lors de la IVe Conférence internationale de psychotechnique de 1927 ; H. Piéron, Mme H. Piéron et H. Laugier : « Étude critique de la valeur sélective du certificat d'études et comparaison de cet examen avec une épreuve par tests, art. cit., p. 499. Cette contribution sera publiée sous le même titre dans la revue du GFEN, Pour l'ère nouvelle, $\mathrm{n}^{\circ} 105$, février 1935, pp. 47-51.

42. Détail intéressant, le Secrétariat général de la commission française pour l'enquête Carnegie sur les examens et les concours, est installé au 41 rue Gay-Lussac, c'est-à-dire à l'INOP, BINOP, n 9, novembre 1932, p. 238. Laugier agit avec le soutien du milieu scientifique auquel il appartient.

43. $\mathrm{H}$. Laugier et $\mathrm{D}$. Weinberg: «Du facteur subjectif dans les notes d'examens ", Année psychologique, XXVIII, 1928, pp. 236-244, et XXXI, 1931 pp. 229-241; H. Laugier et D. Weinberg: "Le facteur subjectif dans les notes d'examen. Deuxième contribution », Année psychologique, XXXI, 1930, pp. 229-241; H. Laugier et D. Weinberg : «Étude comparée des notes d'examens des étudiants et des étudiantes en sciences", Le Travail humain. Revue trimestrielle, mars 1935, pp. 62-81; H. Laugier, Dr Toulouse, D. Weinberg : «Biotypologie et classification scolaire », Extrait des Annales du CNAM, Quatrième série, 1934, n 1, pp. 67-81; H. Laugier et D. Weinberg : Élaboration statistique des données numériques de l'enquête sur la correction des épreuves du bac, Publications de l'Enquête Carnegie sur les examens, 1936.

44. En mai 1931, lors de la conférence internationale d'Eastbourne, le comité français est composé de Barrier (directeur adjoint de l'Enseignement primaire), Bouglé (directeur adjoint de l'ENS), Cope (président du Syndicat des professeurs de lycée), Desclos (sous-directeur de l'Office national des Universités) et Maurain (doyen de la Faculté des sciences). Piéron manifeste sa désapprobation en qualifiant ce comité de réunion "d'administrateurs », BINOP, novembre 1932, p. 238.

45. Piéron évoque en effet «certaines résistances à l'introduction du point de vue psychotechnique, résistances que l'on s'est habitué à rencontrer chez quelques sociologues de tendances littéraires et qui semblent redouter l'introduction de l'esprit expérimental », BINOP, op. cit.

46. BINOP, op. cit. 
47. Examen anthropologique et morphologique; examen sexologique; examen chimique; examen physiologique; examen psychologique; examen de médecine générale; examen psychiatrique.

48. H. Laugier et D. Weinberg : «Le facteur subjectif dans les notes d'examen », in Henri Laugier, Henri Piéron, Mme H. Piéron, Dr E. Toulouse, D. Weinberg: Études docimologiques sur le perfectionnement des examens et concours, Publications du Travail humain, CNAM, 1934, p. 17.

49. H. Laugier et D. Weinberg: «Étude comparée des notes d'examens des étudiants et des étudiantes en sciences », Le Travail humain, mars 1935.

50. Leur étude porte sur 4833 candidats (3 347 étudiants et 1486 étudiantes) entre 1928 et 1930.

51. H. Piéron: "Éducation nouvelle et docimologie», Pour l'ère nouvelle. Revue mensuelle d'éducation nouvelle, décembre 1931, pp. 270-271.

52. Ibid, p. 271.

53. H. Piéron : Examens et docimologie, op. cit., p. 26.

54. H. Piéron : « Chronique docimologique », BINOP, octobre 1933, p. 216.

55. Ibid., p. 218.

56. Cité par H. Piéron : Examens et docimologie, op. cit., p. 27.

57. » Les moyens pratiques d'examiner les connaissances acquises à l'école de base et d'établir une orientation dans les établissements scolaires du degré suivant. Rapport général par L. Dumas ", BINOP, juillet 1934, p. 193.

58. Circulaire du 29 septembre 1924.

59. Les rapports des inspecteurs d'académie insistent sur trois points : la question de l'âge, le problème de la gratuité sur toutes les études, les différences de programme et d'âge.

60. Inspection académique du Doubs au ministre, 3 novembre 1924, F17 13951.

61. Compte rendu des conseils de classes des classes élémentaires ( 4 octobre) du proviseur du lycée de Vesoul à l'inspecteur d'académie de Haute-Saône, 21 octobre 1924, F ${ }^{17} 13951$.

62. BINOP, mars 1934, p. 70.

63. H. Piéron : «Les nouvelles dispositions relatives au certificat d'études », BINOP, juillet-août 1938, p. 148.

64. Jean Zay cité par H. Piéron : «L'examen des examens », BINOP, mars-avril 1937, p. 91.

65. "Sur l'expérience des classes d'orientation », Pour l'ère nouvelle. Revue mensuelle d'éducation nouvelle, décembre 1938, p. 303.

66. J. Fontègne : «Rapport succinct sur la fiche scolaire d'orientation, adopté en Assemblée générale », BINOP, octobre 1937, pp. 190-191.

67. P. Allard: Que faire de nos fils et de nos filles?, Les Éditions de France, 1934, p. 2.

68. » La sélection à l'entrée des universités ", BINOP, novembre 1933, p. 255.

69. Ch. Dietz: La sélection des élèves de l'Enseignement secondaire. Recherche de statistique mathématique et de psychologie expérimentale, Asnières, 1934, p. 16.

70. Ibid., p. 106.

71. Ibid.,p. 110.

72. Commission d'orientation professionnelle du BUIC. Rapport de Madame Chamboulant daté du 12 décembre 1943, AN 63AJ67.

73. Le décret-loi du 24 mai 1938 prévoit une orientation professionnelle et une formation professionnelle obligatoires. Elle s'applique aux élèves quittant l'enseignement primaire et à ceux de l'enseignement technique.

74. Cette enquête sera réalisée en 1943-1944 et publiée en 1950 par l'INED. G. Heuyer, H. Piéron, Mme H. Piéron et A. Sauvy : Le niveau intellectuel des enfants d'âge scolaire (I), Cahier 13 de l'INED, Paris, 1950. 
75. Voir sur ces deux aspects le numéro spécial de 1958 du BINOP intitulé «Études docimologiques de l'enseignement primaire à l'enseignement secondaire » et dirigé par Henri Piéron et Maurice Reuchlin.

\section{RÉSUMÉS}

La docimologie - ou sciences des examens - est apparue dans les années 1920 à l'initiative de scientifiques comme Henri Piéron et Henri Laugier. Elle s'inscrit dans le développement de la psychologie appliquée (travaux d'Alfred Binet et d'Édouard Toulouse) et du mouvement d'éducation nouvelle. Elle a voulu utiliser des méthodes objectives d'évaluation; pour cela, elle s'est attachée à démontrer les lacunes et les insuffisances des examens traditionnels, notamment le certificat d'études primaires et le baccalauréat. Elle s'est appuyée sur l'essor du mouvement d'orientation professionnelle qui proposait de nouveaux objectifs éducatifs et de nouvelles pratiques d'évaluation. Sans connaître une véritable institutionnalisation, la docimologie a clairement influencé les débats sur l'école unique et la mise en œuvre de nouvelles méthodes pédagogiques.

The analysis of tests and exam results appeared in the 20's thanks to scientists like Henri Piéron and Henri Laugier. It was part of the development of applied psychology (works by Alfred Binet and Edouard Toulouse) and of the new education movement. Unbiased appraisal methods were used; in doing so, there were attempts to show the deficiencies and inadequacies of traditional examinations, particularly the primary school diploma and the baccalauréat. The rise of the vocational orientation which offered new teaching objectives and new assessment methods supported that evidence. Though it has never truly been institutionalized, the analysis of tests and exam results has clearly influenced debates on the French one school system and the implementation of new teaching methods.

Die Dozimologie - oder Examenswissenschaft - kam in den 1920er Jahren auf Betreiben von Wissenschaftlern wie Henri Piéron oder Henri Laugier auf. Sie gehört in den Bereich der angewandten Psychologie und der Reformpädagogik; man vergleiche dazu etwa die arbeiten von Alfred Binet oder Edouard Toulouse. Es ging darum, objektivere Bewertungskriterien zu entwickeln und einzusetzen, weshalb man sich darum bemühte, die Probleme und Fehler traditioneller Prüfungsverfahren, etwa im Rahmen der Hauptschulabschluss- oder Abiturprüfung, aufzuzeigen. Man stützte sich auf die aufkommenden Bewegungen zur Berufsfindung, in deren Rahmen neue pädagogische Ziele formuliert und neue Evaluationsmethoden erarbeitet wurden. Ohne dass es zu einer wirklichen Institutionalisierung dieser Praktiken kam, hat die Dozimologie doch die Debatten um die Gesamtschule und die Einführung neuer pädagogischer Konzepte nachhaltig beeinflusst. 
INDEX

Index chronologique : XXe siècle

Index géographique : France

Mots-clés : démocratisation, docimologie, examen, orientation scolaire et professionnelle, psychologie expérimentale

\section{AUTEUR}

\section{JÉRÔME MARTIN}

Doctorant (Université Paris IV)

SHE, URA 1397 